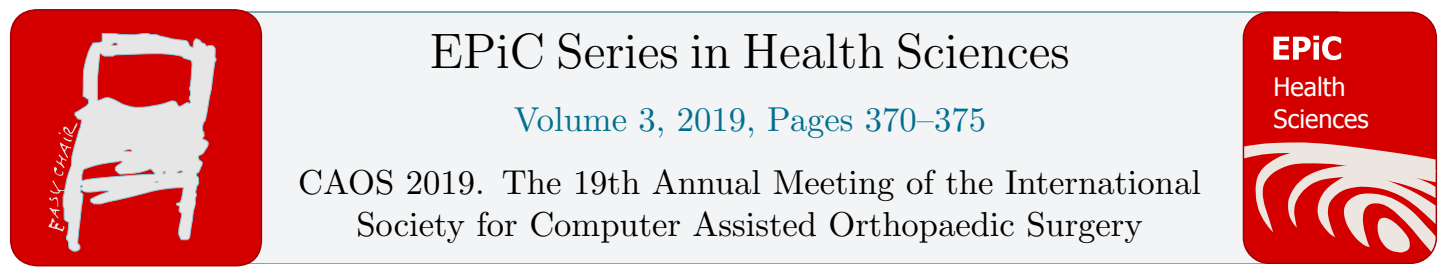

\title{
All-in-one intraoperative 3D imaging and navigation on percutaneous vertebroplasty. Preliminary results on the first 51 patients.
}

\author{
Tonetti $\mathrm{J}^{1}$, MD, PhD; Boudissa $\mathrm{M}^{1}$, MD; Kerschbaumer $\mathrm{G}^{1}$, MD; \\ Prod'homme $\mathrm{M}^{1}$, MD \\ ${ }^{1} \mathrm{CHU}$ Grenoble-Alpes, France \\ JTonetti@chu-grenoble.fr, mboudissa@chu-grenoble.fr, \\ GKerschbaumer@chu-grenoble.fr,
}

\begin{abstract}
Vertebroplasty (VP) is the gold standard for vertebral compression fracture (VCF) treatment. VP could be improved by using intra operative cone bean computed tomography (CBCT) although duration is largely increased. We report first series of 51 patients whom underwent VP integrating intraoperative navigation to CBCT, in order to cut down time consumption. During a 8 month period, 51 patients were prospectively enrolled. The mean age was 71 years old (36-94). There were 17 male and 35 female. We stored 65 VP (14 thoracic and 51 lumbar). After percutaneous pins fixation to the spinous process of the "Butterfly" acquisition reference frame, $180^{\circ}$ rotation of the C-arm CBCT (Surgivisio ${ }^{\circledR}$, eCential, Grenoble France) generated a 3D volume. Optically localized, sterile selfcalibrated trocar was navigated in order to reach the vertebral body center by an unilateral pedicular approach. Cement (Vertaplex HV®, Stryker) was injected under fluoroscopic lateral view by the same machine, using standard 2D X-ray modes. Considering the 38 patients who underwent 1 level VP, mean time procedure was 00:30:09 [00:21:00 to 00:54:00; SD 00:10:23]. The mean DAP was 5,41318 Gy.cm2 and mean exposure duration 3,68 seconds. The mean efficient dose (E) was 1,32 mSv. In all cases cement placement was classified as excellent or mild, without any leakage. This new C-arm CBCT with integrated navigation is efficient to dramatically decrease operating time in comparison to literature (00:46:00 to 00:52:08). The reduction of radiation dose is already demonstrated in literature for navigated $\mathrm{VP}$
\end{abstract}

\section{Introduction}

Vertebroplasty (VP) monitoring by intra operative fluoroscopy is nowadays the gold standard for vertebral compression fracture (VCF) treatment [1]. Procedure is safe, efficient and cost-effectiveness 
[2]. Intra operative 3D imaging coupled to navigation becomes popular in spine surgery [3]. VP could be improved by using intra operative cone bean computed tomography (CBCT) although duration is largely increased 64.8 minutes [36-110] [4].

We report first series of 51 patients whom underwent VP integrating intraoperative navigation to CBCT, in order to cut down time consumption.

\section{Methods}

This is a prospective study of trauma patients with VCF at thoracic and lumbar levels. Patients were included at a single trauma center (Grenoble, France) during a 8 month period (from January to August 2018). The procedure featured general anesthesia on supine position. After percutaneous pins fixation to the spinous process of the "Butterfly" acquisition reference frame, $180^{\circ}$ rotation of the Carm CBCT flat panel (Surgivisio ${ }^{\circledR}$, eCential, Grenoble France), produced 90 or 180 images and generate 3D volume of the spinal area of interest. The acquisition reference frame was then switched to the optical working reference frame. Optically localized, sterile self-calibrated trocar (figure 1) was used as the tool to navigate the trajectory in order to reach the vertebral body center by an unilateral pedicular approach. After check of the calibrating point, automatic axial and sagittal reconstruction views, display on large screen, allow safe, strictly intra osseous, convergent trajectory to the vertebral body (figure 2). Then the cement (Vertaplex HV®, Stryker) was injected, at 8' polymerization, under radiographical lateral view by the same machine, using standard 2D X-ray modes (figure 3 ). Then, a CBCT control of cement filling was done (figure 4).

We stored age, gender, spine level injection on each patient. Time, from pins insertion to the end of the cement injection, was stored in minutes (00:00:00 format). Characteristics of the irradiation were retrieved from the radiology information system providing the total dose area product (DAP) in Gy.cm2. We noted also number of 90-images very low dose CBCT mode and the numbers of 180images low dose CBCT mode. Duration of the full X-ray exposition was stored. The efficient dose (E) in $\mathrm{mSv}$ was calculated from the DAP by application of the conversion $\mathrm{k}$ coefficient of 0,26 for abdominal area and 0,19 for the thoracic area (Source: European commission, RADIATION PROTECTION N ${ }^{\circ}$ 154, European Guidance on Estimating Population Doses from Medical X-Ray Procedures, http://ddmed.eu/_media/background_of_ddm1:rp154.pdf) Quality of the vertebral full filling was noted by poor, mild, excellent. We noted leakage existence (0-1), quality of the displayed images by sufficient or insufficient. 


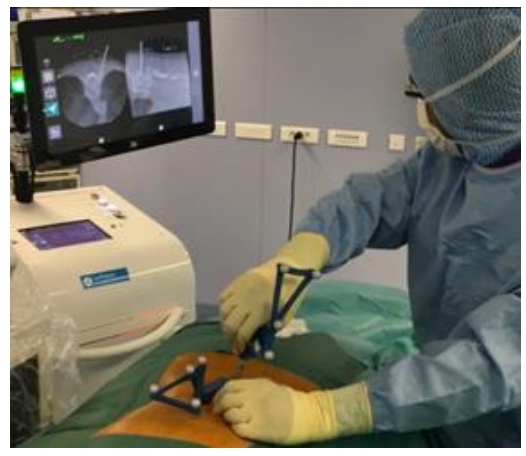

Figure 1 : Optically localized working reference frame and sterile self-calibrated trocar 


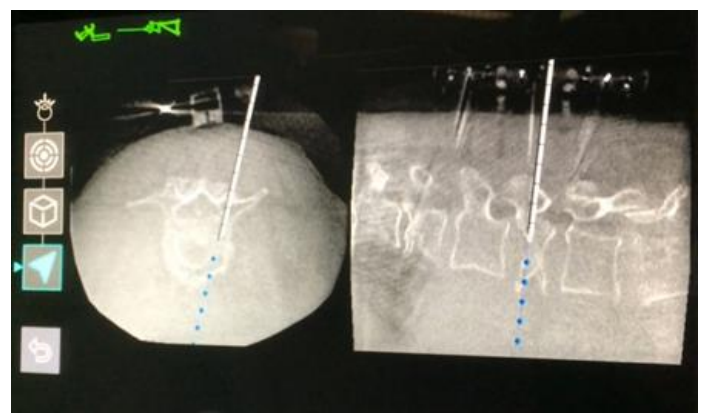

Figure 2 : Navigation of the trajectory on screen display in order to reach the vertebral body center by unilateral pedicular approach

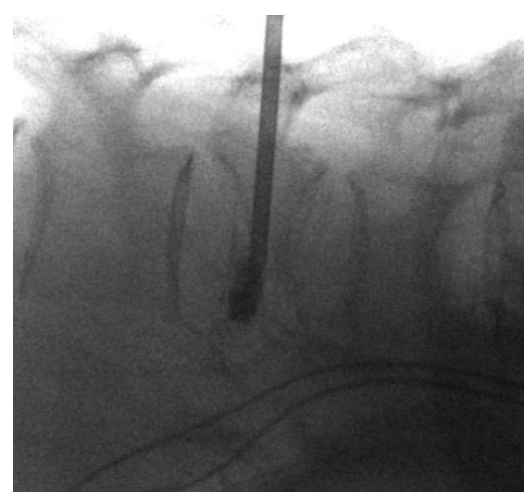

Figure 3 : Radiographical lateral view using standard 2D X-ray mode by the same C-arm machine

\section{Results}

During the so-called period, 51 patients were prospectively enrolled in the study. The mean age was 71 years old (36-94). There were 17 male and 34 female. We stored 65 VP (14 thoracic and 51 lumbar). We noted 38 patients with a single level VP, 12 patients with 2 levels VP and 1 patient with 3 levels VP.

Considering the 38 patients who underwent 1 level VP, mean time for full procedure was 00:30:09 [00:21:00 to 00:54:00; SD 00:10:23]. The mean DAP was 5,41318 Gy.cm2 with mean exposure duration at 3,68 seconds. The CBCT volume was built 24 times with 90 images and 19 times with 180 images. Five patients had double CBCT (2 patients with 90 images, 2 with 180 images and 1 patient with one with 90 images and one with 180 images). The mean efficient dose (E) was 1,32 mSv for 1 level VP. In all cases cement was classified excellent in 25 cases and mild in 13 cases, located at the center of the body, without any leakage in the intervertebral space, foramina or the vertebral canal. Quality of the image was noticed sufficient to perform a secure trocar placement in all cases. 


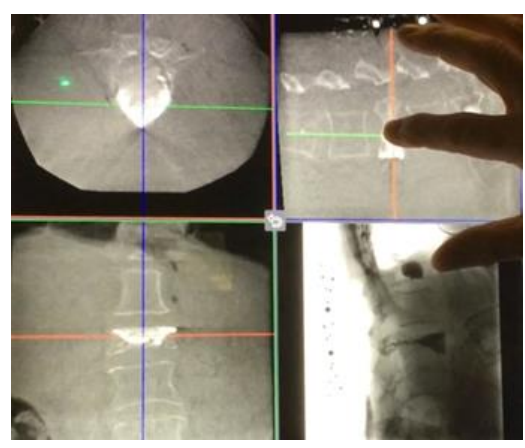

Figure 4 : intraoperative cone bean computed tomography (CBCT) control of cement filling

\section{Discussion}

In our experience this new C-arm CBCT with integrated navigation is efficient to dramatically decrease operating time. Tam et al. using Axiom Artis dTA VB31 (Siemens) found mean operating time 00:64:48 64 [range 36-110] [4]. Ruatti et al. using 3D intraoperative Arcadis Orbic system (Siemens) found a mean operative time by level 00:46:00 $( \pm 25.13)$ [5]. Shin and Jeong using SIREMOBIL isoC3D (Siemens) found a mean operating time 00:52:08 [6]. Our 30 minutes time was consistent for all categories of surgeons in our academic hospital (resident, assistant and senior). It indicates that the step-by-step process, included in the system, helps to standardize the surgical technique.

The reduction of radiation dose is already demonstrated in literature for navigated vertebroplasty [7, 8]. Comparing the results is difficult without having full details on the series. However, our experience tends to show that improvement is still possible by using recent technologies.

We also noticed at the beginning of each procedure a high degree of confidence and predictability from the staff in the time. This brings calm and relax atmosphere in the OR.

The pin fixation of the reference frame is percutaneous. Stability is enhanced by fixation on 2 or 3 adjacent vertebrae spinous process. Using a conventional clamp for reference fixation would not be appropriate for vertebroplasty. In addition, using this special butterfly device, it is not necessary to stop patient breathing during 3D image acquisition, which is also an important element of the simplified process with this novel device. 


\section{Conclusion}

Contrary to our previous experience with 3D imaging using CBCT and coupled with conventional navigation, this paper reports very positive feedback about using a novel CBCT imaging device, integrating navigation. For the studied vertebroplasty indication, an average surgical time of about 30 minutes was reported, which in our case, is less than our average time using conventional fluoroscopy.

\section{References}

1. Klazen CA, Lohle PN, de Vries J, Jansen FH, Tielbeek AV, Blonk MC, Venmans A, van Rooij WJ, Schoemaker MC, Juttmann JR, Lo TH, Verhaar HJ, van der Graaf Y, van Everdingen KJ, Muller AF, Elgersma OE, Halkema DR, Fransen H, Janssens X, Buskens E, Mali WP. Vertebroplasty versus conservative treatment in acute osteoporotic vertebral compression fractures (Vertos II): an open-label randomised trial. Lancet. 2010 Sep 25;376(9746):1085-92

2. Wang H, Sribastav SS, Ye F, Yang C, Wang J, Liu H, Zheng Z. Comparison of Percutaneous Vertebroplasty and Balloon Kyphoplasty for the Treatment of Single Level Vertebral Compression Fractures: A Meta-analysis of the Literature. Pain Physician. 2015 MayJun;18(3):209-22

3. Adamczak SE, Bova FJ, Hoh DJ. Intraoperative 3D Computed Tomography: Spine Surgery. Neurosurg Clin N Am. 2017 Oct;28(4):585-594

4. Tam AL, Mohamed A, Pfister M, Chinndurai P, Rohm E, Hall AF, Wallace MJ. C-arm cone beam computed tomography needle path overlay for fluoroscopic guided vertebroplasty. Spine (Phila Pa 1976). 2010 May 1;35(10):1095-9

5. Ruatti S, Dubois C, Chipon E, Kerschbaumer G, Milaire M, Moreau-Gaudry A, Tonetti J, Merloz P. Interest of intraoperative 3D imaging in spine surgery: a prospective randomized study. Eur Spine J, 2016; 25(6):1738-44

6. Shin JH, Jeong JH. Preliminary report of three-dimensional reconstructive intraoperative C-arm in percutaneous vertebroplasty. J Korean Neurosurg Soc. 2012 Feb;51(2):120-3

7. Izadpanah K, Konrad G, Südkamp NP, Oberst M. Computer navigation in balloon kyphoplasty reduces the intraoperative radiation exposure. Spine (Phila Pa 1976). 2009 May 20;34(12):13259

8. Lee YS, Lee HK, Cho JH, Kim HG. Analysis of radiation risk to patients from intra-operative use of the mobile X-ray system (C-arm). J Res Med Sci. 2015 Jan;20(1):7-12. 